\title{
Aggregation-related non-photochemical quenching in the photosynthetic membrane
}

Jevgenij Chmeliov, ${ }^{\dagger, \ddagger}$ Andrius Gelzinis, ${ }^{\dagger}$, Marius Franckevičius, ${ }^{\ddagger}$ Marijonas Tutkus, ${ }^{\ddagger}$ Francesco Saccon, ${ }^{\text {II }}$ Alexander V. Ruban, ${ }^{*, \mathbb{I}}$ and Leonas Valkunas, ${ }^{*},+$

${ }^{\dagger}$ Institute of Chemical Physics, Faculty of Physics, Vilnius University, Saulètekio Ave. 9, LT-10222 Vilnius, Lithuania

${ }^{\ddagger}$ Department of Molecular Compound Physics, Center for Physical Sciences and Technology, Saulètekio Ave. 3, LT-10257 Vilnius, Lithuania

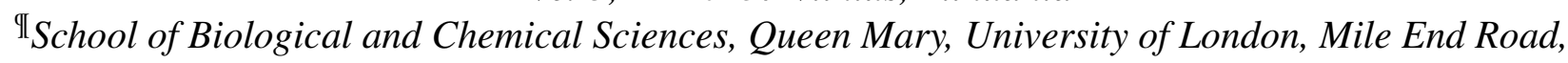
London E1 4NS, U.K.

*Corresponding authors: a.ruban@qmul.ac.uk, leonas.valkunas@ff.vu.lt

\section{Supplementary Information}




\section{Methods}

Plant material and growth conditions. Arabidopsis thaliana Col-0 ecotype (WT), and PsbSover-expressing (L17) plants were used in this study. Seeds were sterilized in $50 \%$ ethanol, $0.1 \%$ Triton X-100 for 1 minute and vernalized at $4{ }^{\circ} \mathrm{C}$ for 2 days before sowing. Seedlings were grown under $90-110 \mu \mathrm{mol}$ photons $\mathrm{m}^{-2} \mathrm{~s}^{-1}$ for 2 weeks and then transferred to a growth chamber at $22{ }^{\circ} \mathrm{C}$, under $180 \mu \mathrm{mol}$ photons $\mathrm{m}^{-2} \mathrm{~s}^{-1}$ and $10 \mathrm{~h}$ photoperiod conditions. Treatment with the antibiotic lincomycin (Sigma-Aldrich) ${ }^{1,2}$ was started at the rosette stage (50\% of the final size). Plants were watered three times a week with increasing concentration of lincomycin $(0.1-0.6 \mathrm{~g} / \mathrm{L})$ dissolved in water, until the ratio $F_{\mathrm{v}} / F_{\mathrm{m}}$ (here $F_{\mathrm{v}}$ is variable fluorescence, $F_{\mathrm{v}}=F_{\mathrm{m}}-F_{0}$ ) reached 0.35 or lower.

\section{Chloroplasts isolation and characterisation.}

Whole plants were dark adapted for 45 minutes before chloroplasts extraction. Intact chloroplasts were prepared as described in Ref. 3 with modifications. Briefly, fresh leaf material was homogenised in grinding buffer $(0.33 \mathrm{M}$ sorbitol, $40 \mathrm{mM}$ D-isoascorbate, $10 \mathrm{mM} \mathrm{Na} 4 \mathrm{P}_{2} \mathrm{CO}_{7}$, $2.5 \mathrm{mM}$ EDTA, $5 \mathrm{mM} \mathrm{MgCl} 2$; $\mathrm{pH}$ 6.5) and filtered through four layers of muslin, followed by four layers of muslin and one layer of cotton wool. Filtrate was centrifuged for $2 \mathrm{~min}$ at $4000 \mathrm{RCF}$. The pellet was then resuspended in a few drops of resuspension buffer (RB; $0.33 \mathrm{M}$ sorbitol, $10 \mathrm{mM}$ $\mathrm{KCl}, 2.5 \mathrm{mM}$ EDTA, $5 \mathrm{mM} \mathrm{MgCl} 2$; pH 7.6), layered on top of a Percoll density gradient $(50 \%$ Percoll (Sigma-Aldrich) - $50 \%$ RB double osmotic) and centrifuged for $6 \mathrm{~min}$ at $4000 \mathrm{RCF}$. Intact chloroplasts were retrieved in the pellet and resuspended in RB for measurements. Their intactness was evaluated by direct observation at the light microscope and by comparing $F_{\mathrm{v}} / F_{\mathrm{m}}$ to whole leaf measurements. Total chlorophyll content was determined by pigment extraction in $80 \%$ acetone, as described before. ${ }^{4}$

Chlorophyll fluorescence analysis. Chlorophyll fluorescence was measured with a Dual PAM 100 measuring system (Walz, Germany). Chloroplasts were resuspended in $1.6 \mathrm{ml}$ of RB $(50 \mu \mathrm{g} / \mathrm{ml}$ total chlorophyll). Actinic light $(2000 \mu \mathrm{mol}$ photons $\mathrm{m}^{-2} \mathrm{~s}^{-1}$ ) was provided by arrays of $635-\mathrm{nm}$ LEDs for 40 minutes and the temperature was set to constant $20{ }^{\circ} \mathrm{C}$. Saturating pulses were applied at fixed intervals to determine the NPQ amplitude, defined as $\left(F_{\mathrm{m}}-F_{\mathrm{m}}^{\prime}\right) / F_{\mathrm{m}}^{\prime}$. After $40 \mathrm{~min}$ utes of illumination with high light, the maximum NPQ reached was $\sim 7.8$ for WT and $\sim 10.5$ for L17 chloroplasts. The low amount of RCII in our samples ensures that in this stabilized quenched state NPQ is dominated by the energy-dependent quenching ( $\mathrm{qE}$ ) while the photoinhibitory part is negligible. Samples were frozen in liquid nitrogen and stored for further measurements. The intactness of the sample and the rearrangements of LHCs throughout the inductions were evaluated by $77 \mathrm{~K}$ fluorescence emission measurements (while excited at $435 \mathrm{~nm}$ ) using a Jobin Yvon FluoroMax-3 fluorometer. No blue-shifted signal due to free Chls from the damaged complexes was registered.

\section{Sample preparation for the time-resolved FL} measurements. To reduce ice formation during sample freezing with the cryostat, which otherwise creates too much scattering, the surface of the cuvette was modified with a PEG monolayer. Briefly, demountable cell (fused silica cell of $0.2 \mathrm{~mm}$ optical path, $22 \mathrm{~mm}$ diameter, 124-0.2-40, Hellma Analytics) was cleaned by washing with detergent solution (1104, Alconox) supplemented with an anti-foaming agent (YC39.1, Carl-Roth), DI water (Labostar, Siemens) and then etched for $5 \mathrm{~min}$ with air plasma (high power mode, PDC-002, Harric Plasma). Next, the surface of the cleaned cuvette was silanized for 5 minutes using $\mathrm{N}$ (2-Aminoethyl)-3-aminopropyltrimethoxysilane (819172, Merck Group) 1 \% (v/v) solution in Methanol (822283, Merck Group) supplemented with $5 \%$ (v/v) acetic acid (320099, SigmaAldrich). After silanization the cuvette was washed with methanol, DI water, then dried and placed inside upwards on a pipette tip rack. Further, the surface was PEGylated using $2 \mathrm{mg} / 100 \mu \mathrm{l}$ methoxy-PEG5000-SVA (Lyasan Bio, USA) solution in sodium bicarbonate buffer $(\mathrm{pH} 8.5$, HN01.1, Carl-Roth). $40 \mu \mathrm{l}$ drop of the mPEGSVA solution was placed on the silanized cuvette and then the second half of the cuvette was placed on this drop (sandwich assembly). PEGylation reaction was performed overnight, then the cuvette was disassembled, cleaned by washing with DI water and dried using nitrogen gas. The thylakoid solution was diluted with glycerol (G5516, SigmaAldrich) so that the final sample was $60 \%(\mathrm{v} / \mathrm{v})$ glycerol solution. $35 \mu \mathrm{l}$ of this sample was placed 
into the cuvette with PEGylated surface. The cuvette was glued firmly by making a thin vacuum grease (Down Corning high vacuum grease) layer along its perimeter.

Experimental measurements. Time-resolved fluorescence dynamics of the samples were measured by means of Hamamatsu C5680 streak camera with either M5675 (synchroscan) or M5677 (single-sweep) module coupled to a spectrometer. Femtosecond $\mathrm{Yb}: \mathrm{KGW}$ oscillator (Pharos, Light Conversion Ltd) with a frequency doubler (HIRO, Light Conversion Ltd) producing $515 \mathrm{~nm}$ sub-100-fs pulses at a $76 \mathrm{MHz}$ repetition rate was employed, and a pulse picker was used to reduce the repetition rate to $20 \mathrm{kHz}$ in the single-sweep mode for nanosecond timescales. The beam was attenuated down to about $1 \mathrm{pJ}$ (excitation energy density of about $\left.24 \mathrm{~nJ} \mathrm{~cm}^{-2}\right)(76 \mathrm{MHz}$ repetition rate, picosecond timescales) or $100 \mathrm{pJ}$ (excitation energy density of about $\left.0.5 \mathrm{~nJ} \mathrm{~cm}^{-2}\right)(20 \mathrm{kHz}$ repetition rate, nanosecond timescales) per pulse and focused into about $80 \mu \mathrm{m}$ spot on the sample. The temporal resolution of the whole system was around $10 \mathrm{ps}$ (full width at half maximum) for the synchroscan mode and $\sim 100$ ps for the single-sweep mode. A liquid helium cold finger cryostat (Janis CCS-100/204) was used for low temperature fluorescence measurements.

Data analysis. The measured time-resolved fluorescence spectra $F(\lambda, t)$ of LHCII trimers and aggregates under various temperatures were analysed in terms of multivariate curve resolution ${ }^{5}$ using a multiplicative update algorithm, ${ }^{6}$ similarly as in our previous work. ${ }^{7}$ For each dataset, the factorisation according to

$$
F(\lambda, t)=\sum_{n=1}^{N} S_{n}(\lambda) K_{n}(t)+E(\lambda, t) .
$$

(or, in matrix form, $\mathbf{F}=\mathbf{S} \cdot \mathbf{K}+\mathbf{E}$, were the residual matrix $\mathbf{E}$ is minimised) was carried out for 5000 times starting from random non-negative initial matrices $\mathbf{S}$ and $\mathbf{K}$. As shown in Supplementary Figs. S2-S5, all the experimental data is perfectly reproduced at the initial time delays, but some discrepancies appear at longer time delays, probably due to population of some additional conformational states of the pigment-protein complexes or the heterogeneity of the thylakoid membranes.
The origins of the latter might be due to the presence of minor and major antenna complexes, also possibly some LHCI complexes from PSI antenna or formation of the variously sized clusters of the pigment-proteins.

To address any possible differences in the structural organization of pigment-proteins within the thylakoids, the FL decay kinetics at $680 \mathrm{~nm}$, obtained at $270 \mathrm{~K}$ temperature for WT and L17 samples under NPQ conditions and LHCII were further analyzed in terms of our recently developed fluctuating antenna model that was proved to successfully describe excitation energy dynamics in the various disordered light-harvesting systems prone to some structural fluctuations. ${ }^{8-10}$ In this model, the usually observed multi-exponential fluorescence decay kinetics are described by using only 3 parameters - the effective fractional dimensionality $d$ that reflects structural organization of the system, the timescale factor $\eta=$ $2 d \tau_{\mathrm{h}}\left(\left\langle N_{\mathrm{q}}\right\rangle / N\right)^{-2 / d}$ that relates the mean excitation inter-complex transfer time $\tau_{\mathrm{h}}$ with the mean relative concentration of traps $\left\langle N_{\mathrm{q}}\right\rangle / N$, and intrinsic excitation dissipation rate due to radiative or non-radiative relaxation to the ground state $k_{\text {dis. }}$. The latter rate was fixed to a well-known value of $k_{\text {dis }}^{-1}=3.5 \mathrm{~ns},{ }^{7}$ leaving just two undefined model parameters $(d$ and $\eta$ ). The obtained fits together with the corresponding model parameters for room-temperature FL kinetics in both WT and L17 light-pretreated thylakoids are shown in Supplementary Fig. S8a. We can see that in both cases, the structural mutual arrangements of the pigment-protein complexes within the thylakoid should be very similar: both systems exhibited the same dimensionality $d \approx 1.75$, obviously meaning 2-dimensional manner of excitation energy transfer as well as the existence of some void regions and lack of connectivity between some antenna parts. This value is rather similar to $d=1.67$ that was obtained for LHCII aggregates. ${ }^{7}$ Moreover, by assuming mean inter-complex excitation energy transfer of $\tau_{\mathrm{h}}=25 \mathrm{ps},{ }^{7,8,11,12}$ from the second model parameter $\eta$ we can evaluate the mean concentration of traps. As shown in Supplementary Fig. S8b, we get that on average under NPQ conditions $\sim 10 \%$ of the antenna complexes in the WT thylakoids are in the quenched conformation-roughly the same amount was also obtained previously for LHCII aggregates ${ }^{7}$ (at $270 \mathrm{~K}$ temperature, the FL kinetics in LHCII aggregates ex- 
hibited similar decay rate as in the light-adapted thylakoid membranes). Meanwhile, in the PsbS over-expressed L17 mutant the relative concentration of traps is indeed slightly higher, as suggested in the main text, and reaches $\sim 13 \%$.

\section{References}

(1) Belgio, E.; Johnson, M. P.; Jurić, S.; Ruban, A. V. Higher Plant Photosystem II Light-Harvesting Antenna, Not the Reaction Center, Determines the ExcitedState Lifetime-Both the Maximum and the Nonphotochemically Quenched. Biophys. J. 2012, 102, 2761-2771.

(2) Ware, M. A.; Giovagnetti, V.; Belgio, E.; Ruban, A. V. PsbS Protein Modulates Non-Photochemical Chlorophyll Fluorescence Quenching in Membranes Depleted of Photosystems. J. Photochem. Photobiol. B 2015, 152, 301-307.

(3) Crouchman, S.; Ruban, A.; Horton, P. PsbS Enhances Nonphotochemical Fluorescence Quenching in the Absence of Zeaxanthin. FEBS Lett. 2006, 580, 2053-2058.

(4) Porra, R. J.; Thompson, W. A.; Kriedemann, P. E. Determination of Accurate Extinction Coefficients and Simultaneous Equations for Assaying Chlorophylls $a$ and $b$ Extracted with Four Different Solvents: Verification of the Concentration of Chlorophyll Standards by Atomic Absorption Spectroscopy. Biochim. Biophys. Acta, Bioenerg. 1989, 975, 384-394.

(5) Lawton, W. H.; Sylvestre, E. A. Self Modeling Curve Resolution. Technometrics 1971, 13, 617-633.

(6) Berry, M. W.; Browne, M.; Langville, A. N.; Pauca, V. P.; Plemmons, R. J. Algorithms and Applications for Approximate Nonnegative Matrix Factorization. Comput. Stat. Data Anal. 2007, 52, 155-173.

(7) Chmeliov, J.; Gelzinis, A.; Songaila, E.; Augulis, R.; Duffy, C. D. P.; Ruban, A. V.; Valkunas, L. The Nature of Self-Regulation in Photosynthetic Light-Harvesting Antenna. Nat. Plants 2016, 2, 16045.
(8) Chmeliov, J.; Trinkunas, G.; van Amerongen, H.; Valkunas, L. Light Harvesting in a Fluctuating Antenna. J. Am. Chem. Soc. 2014, 136, 8963-8972.

(9) Chmeliov, J.; Trinkunas, G.; van Amerongen, H.; Valkunas, L. Excitation Migration in Fluctuating Light-Harvesting Antenna Systems. Photosynth. Res. 2016, 127, 49-60.

(10) Trinkunas, G.; Chmeliov, J. Fluctuating Antenna Model: Applications and Prospects. Lith. J. Phys. 2018, 58, 379-390.

(11) Barzda, V.; Gulbinas, V.; Kananavicius, R.; Cervinskas, V.; van Amerongen, H.; van Grondelle, R.; Valkunas, L. Singlet-Singlet Annihilation Kinetics in Aggregates and Trimers of LHCII. Biophys. J. 2001, 80, 2409-2421.

(12) Bennett, D. I. G.; Amarnath, K.; Fleming, G. R. A Structure-Based Model of Energy Transfer Reveals the Principles of Light Harvesting in Photosystem II Supercomplexes. J. Am. Chem. Soc. 2013, 135, 91649173. 

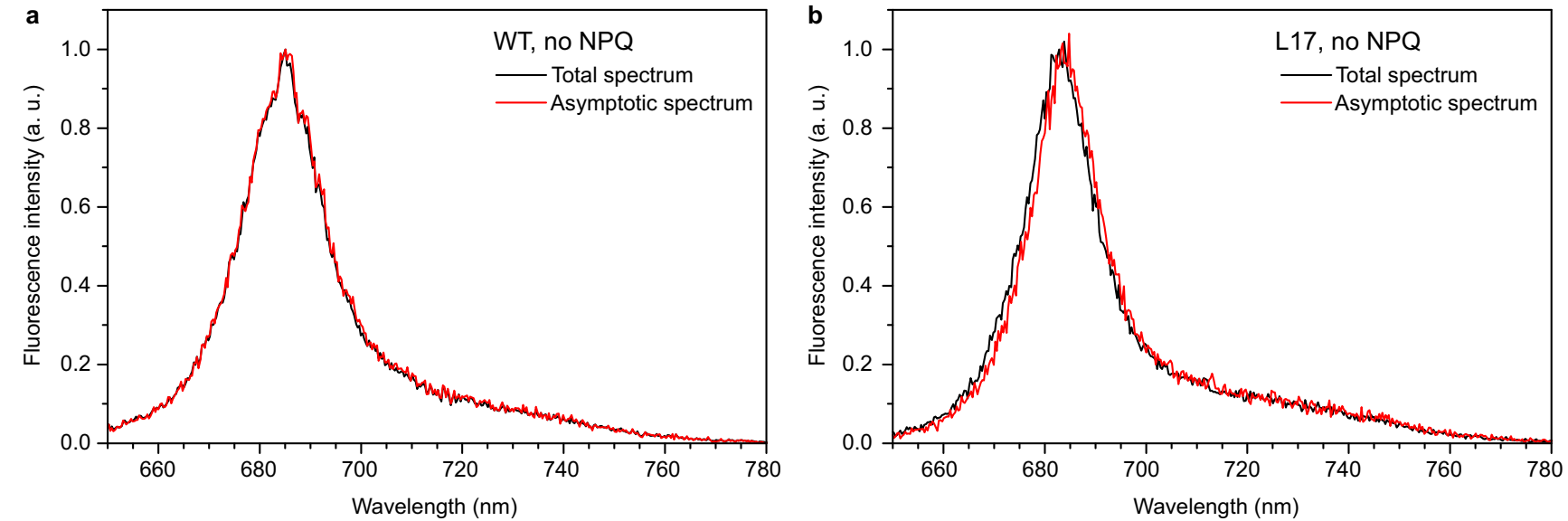

Figure S1. Comparison of the total integrated and asymptotic (integrated over 1-8 ns time window and thus completely lacking any possible signal from PSI) room-temperature FL spectra of the lincomycintreated dark-adapted chloroplasts from the WT (a) and L17 mutant (b). 

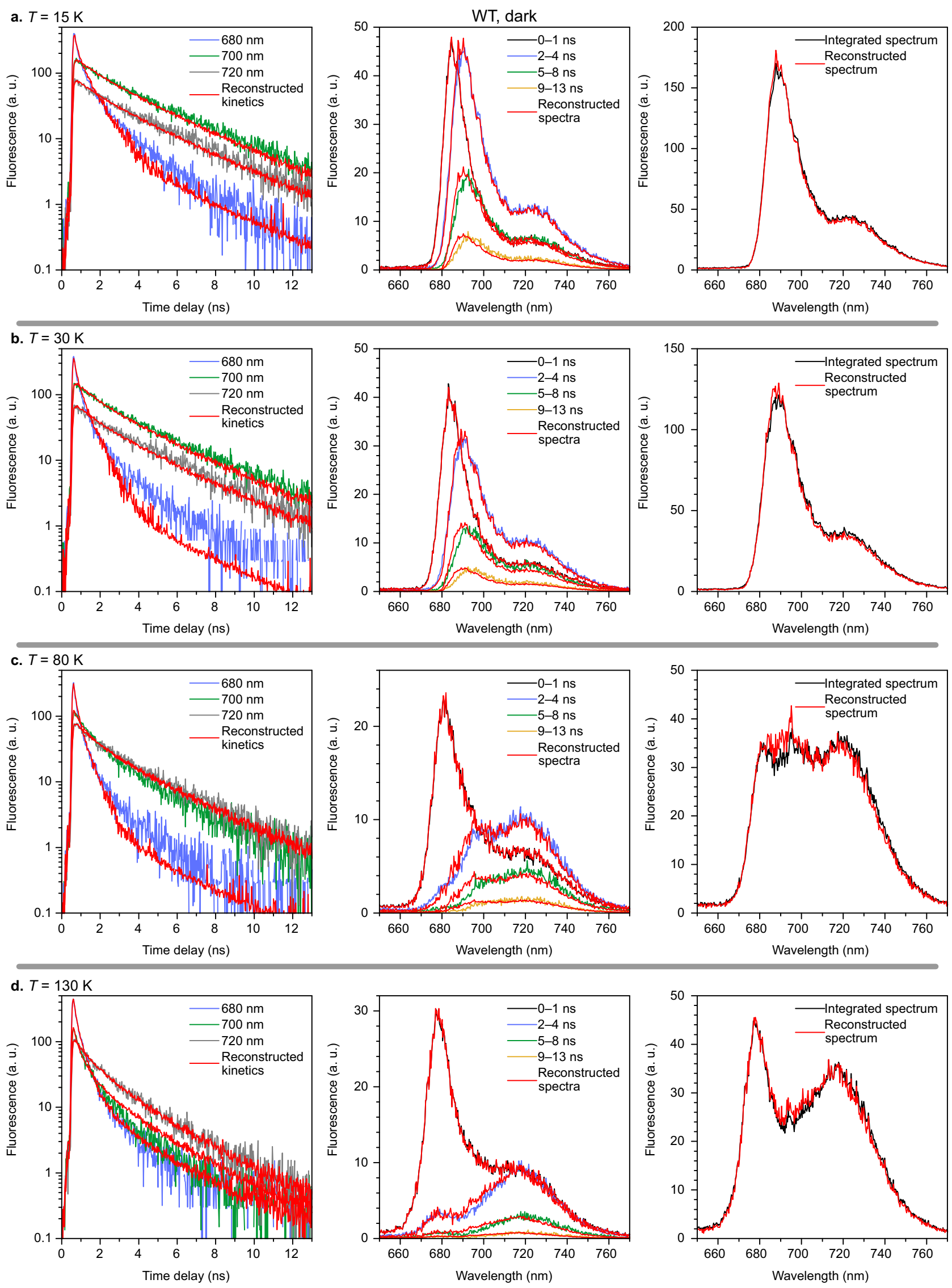

Figure S2. Fluorescence decay kinetics at different wavelengths (left), fluorescence spectra at different time delays (middle), and total integrated fluorescence spectra (right), obtained during the streak camera measurements of dark-adapted chloroplasts from WT lincomycin-pretreated Arabidopsis thaliana at $15 \mathrm{~K}$ (a), $30 \mathrm{~K}(\mathbf{b}), 80 \mathrm{~K}$ (c) and $130 \mathrm{~K}$ (d) temperatures. Red lines in all panels illustrate corresponding fluorescence kinetics and spectra, reconstructed from the 2-state multivariate curve resolution analysis. 

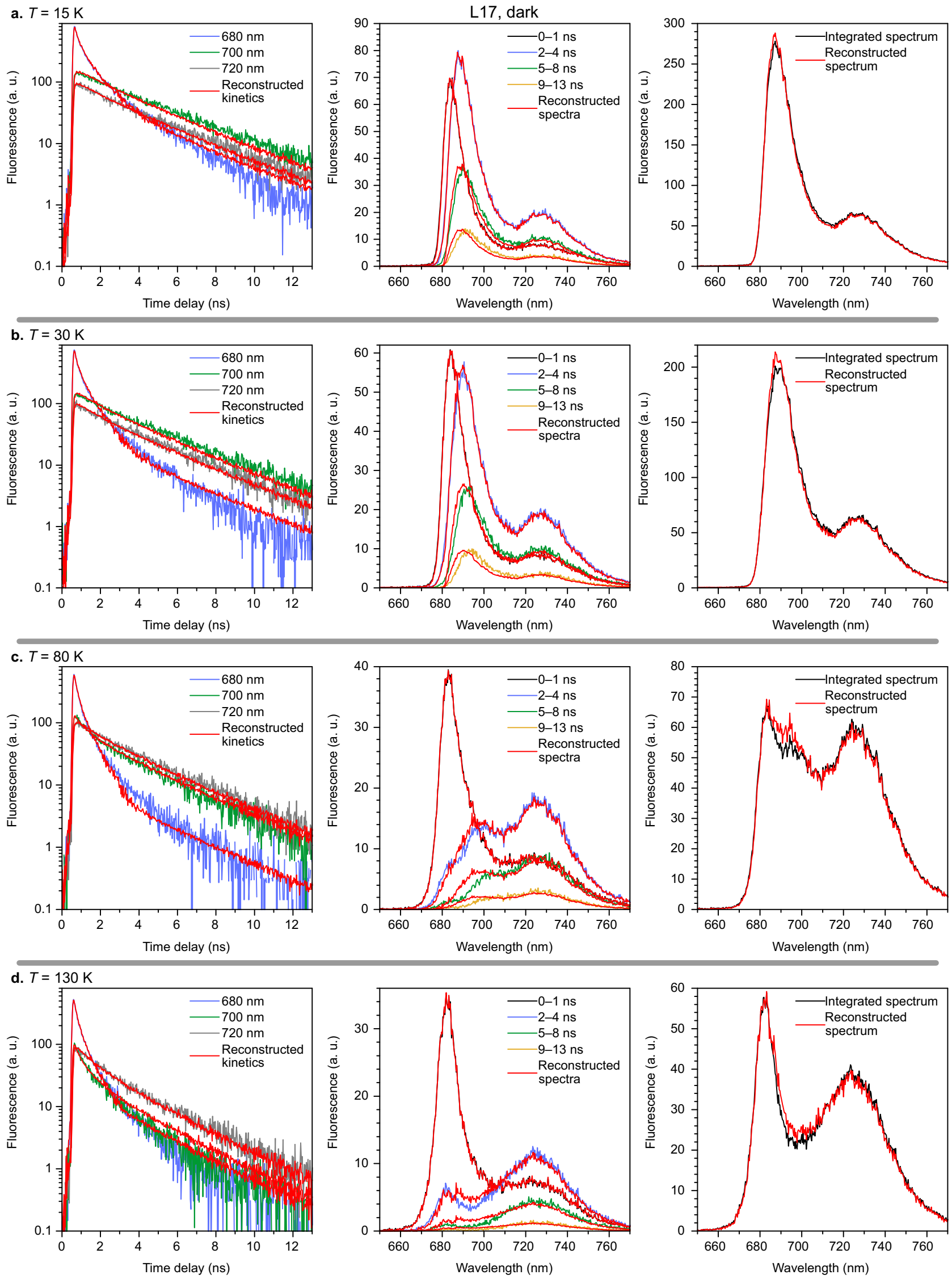

Figure S3. Fluorescence decay kinetics at different wavelengths (left), fluorescence spectra at different time delays (middle), and total integrated fluorescence spectra (right), obtained during the streak camera measurements of dark-adapted chloroplasts from lincomycin-pretreated Arabidopsis thaliana L17 mutant at $15 \mathrm{~K}(\mathbf{a}), 30 \mathrm{~K}(\mathbf{b}), 80 \mathrm{~K}(\mathbf{c})$ and $130 \mathrm{~K}(\mathbf{d})$ temperatures. Red lines in all panels illustrate corresponding fluorescence kinetics and spectra, reconstructed from the 2-state multivariate curve resolution analysis. 

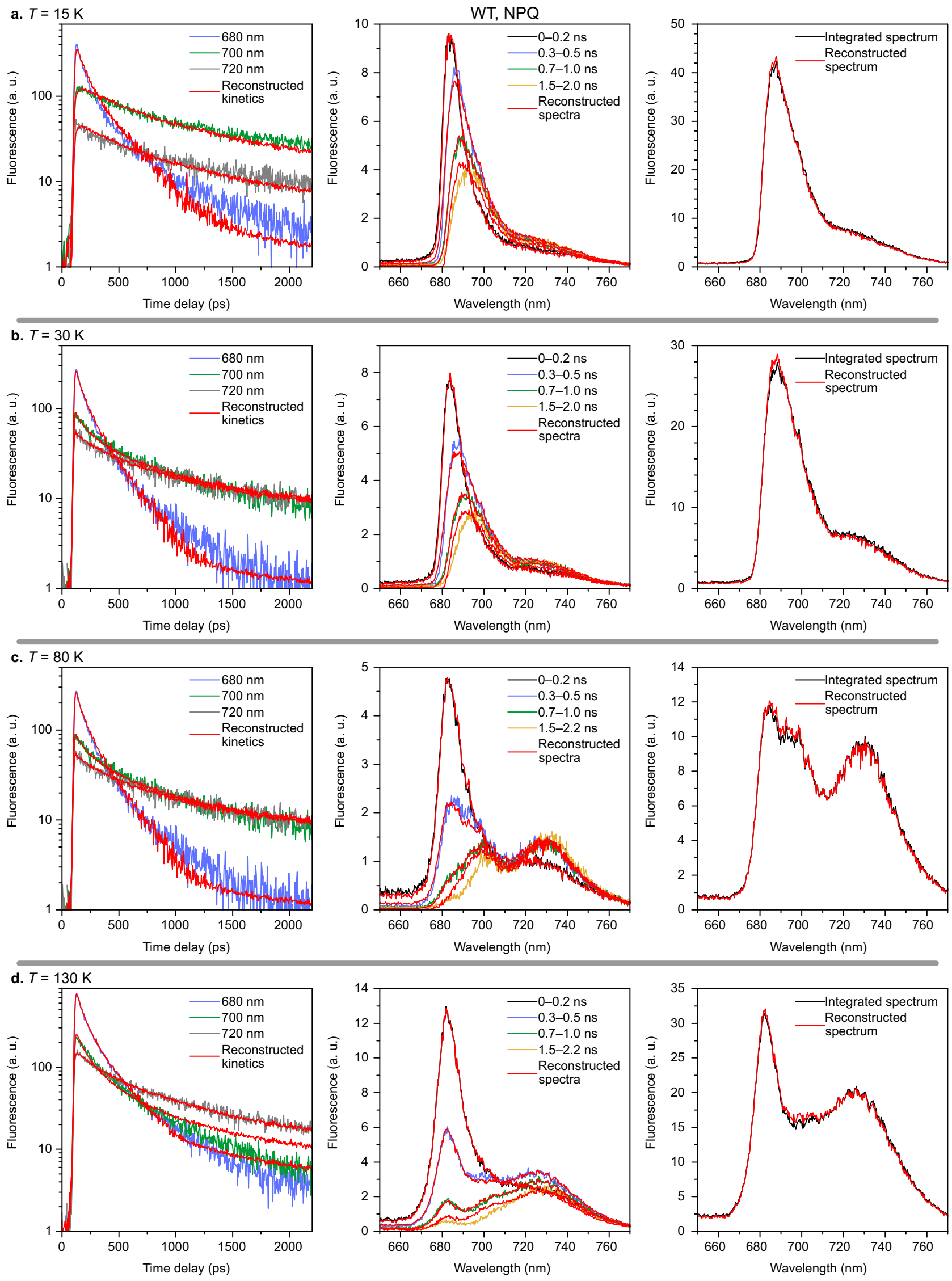

Figure S4. Fluorescence decay kinetics at different wavelengths (left), fluorescence spectra at different time delays (middle), and total integrated fluorescence spectra (right), obtained during the streak camera measurements of light-adapted chloroplasts from WT lincomycin-pretreated Arabidopsis thaliana at $15 \mathrm{~K}$ (a), $30 \mathrm{~K}(\mathbf{b}), 80 \mathrm{~K}$ (c) and $130 \mathrm{~K}$ (d) temperatures. Red lines in all panels illustrate corresponding fluorescence kinetics and spectra, reconstructed from the 2-state multivariate curve resolution analysis. 

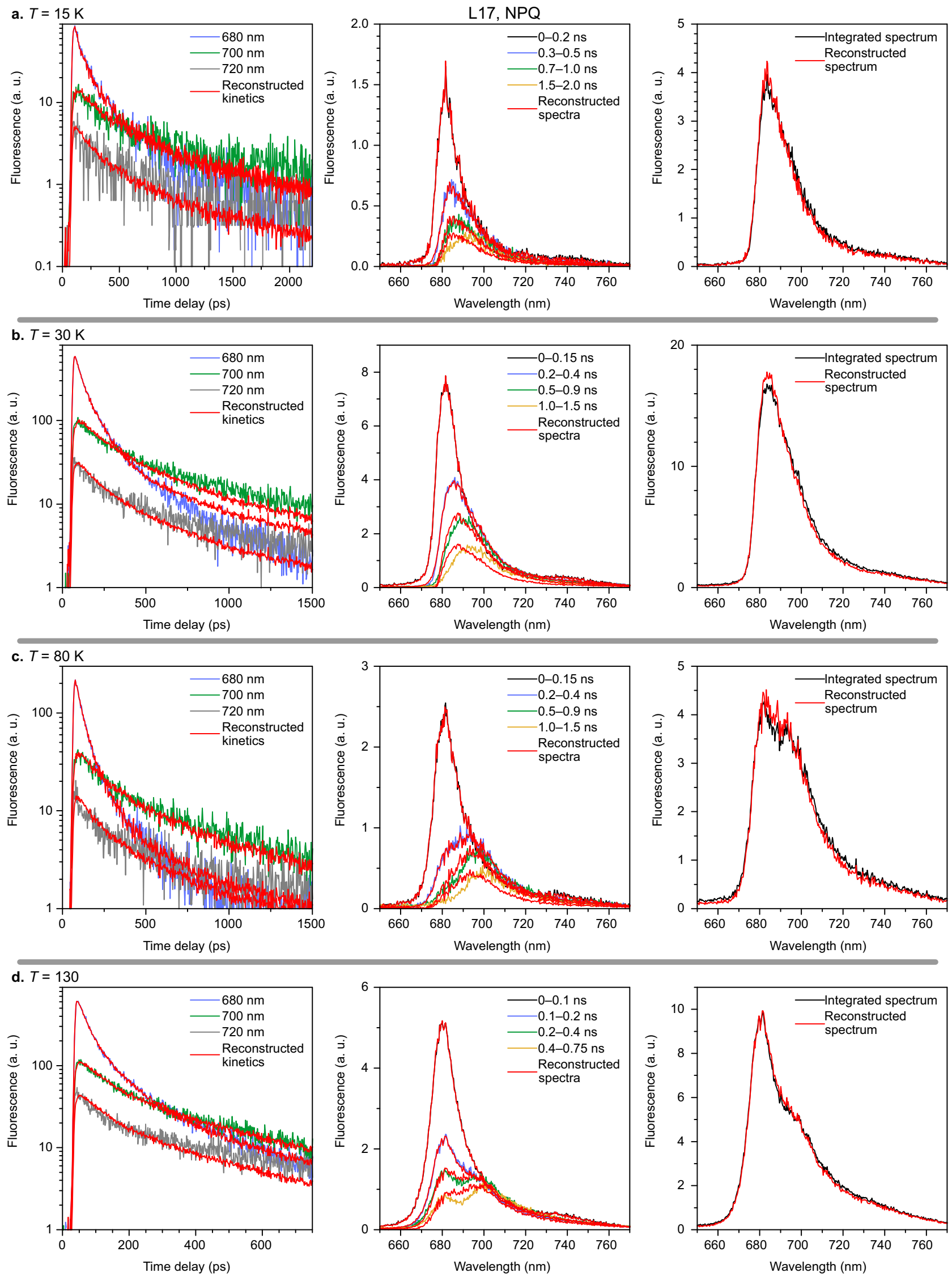

Figure S5. Fluorescence decay kinetics at different wavelengths (left), fluorescence spectra at different time delays (middle), and total integrated fluorescence spectra (right), obtained during the streak camera measurements of light-adapted chloroplasts from lincomycin-pretreated Arabidopsis thaliana L17 mutant at $15 \mathrm{~K}(\mathbf{a}), 30 \mathrm{~K}(\mathbf{b}), 80 \mathrm{~K}(\mathbf{c})$ and $130 \mathrm{~K}(\mathbf{d})$ temperatures. Red lines in all panels illustrate corresponding fluorescence kinetics and spectra, reconstructed from the 2-state multivariate curve resolution analysis. 

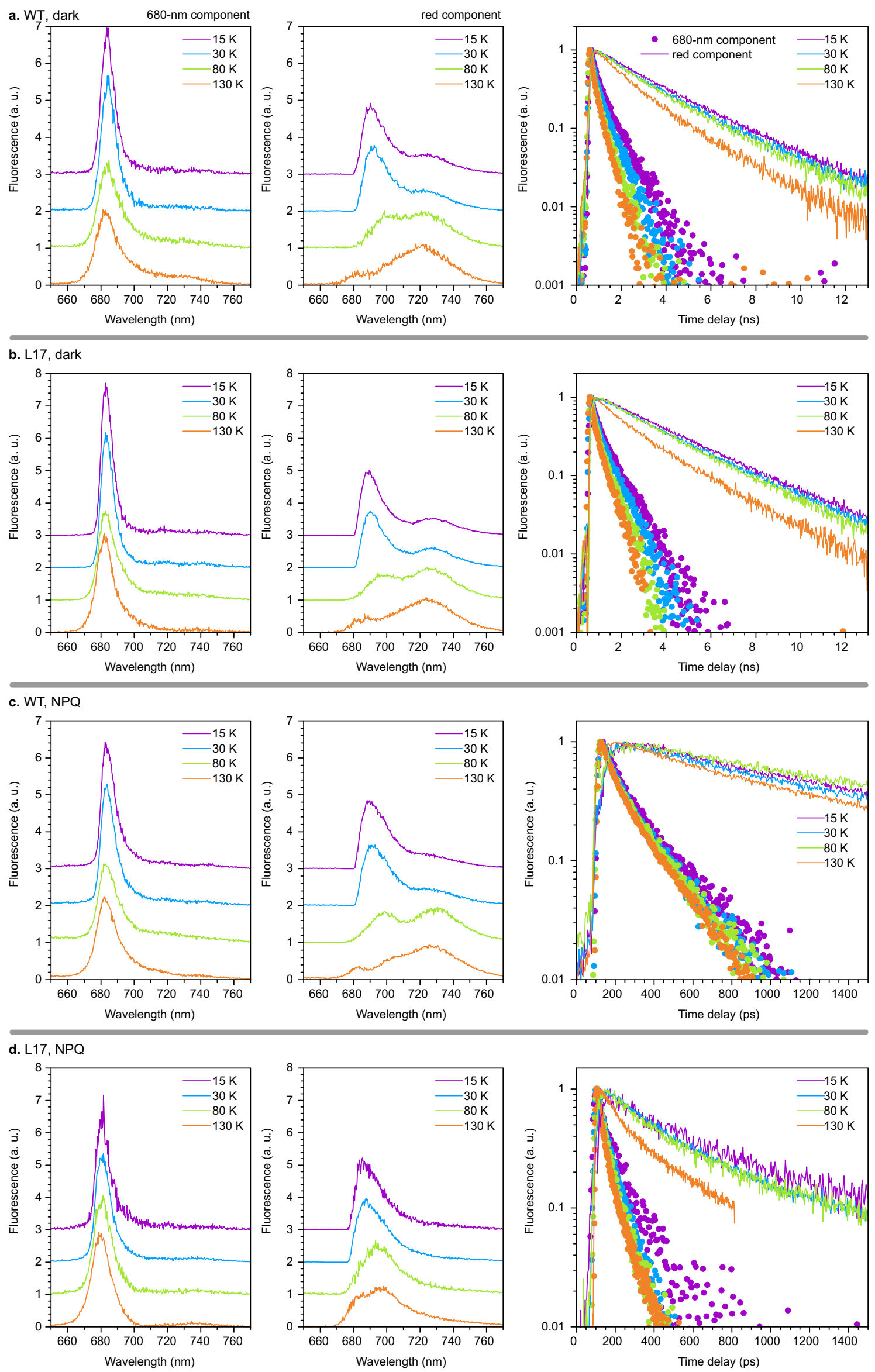

Figure S6. Summarized results of the 2-state multivariate curve resolution analysis of the FL from chloroplasts from different samples (a-d) at various temperatures. Left, temperature-dependent fluorescence spectra of the dominating 680-nm-emitting state. Middle, temperature-dependent fluorescence spectra of the red-emitting states. All fluorescence spectra are normalized to the area below them. Right, normalized temperature-dependent fluorescence decay kinetics of the 680-nm-emitting (dots) and red-emitting (lines) states and spectra at different temperatures are shifted vertically for clarity. 

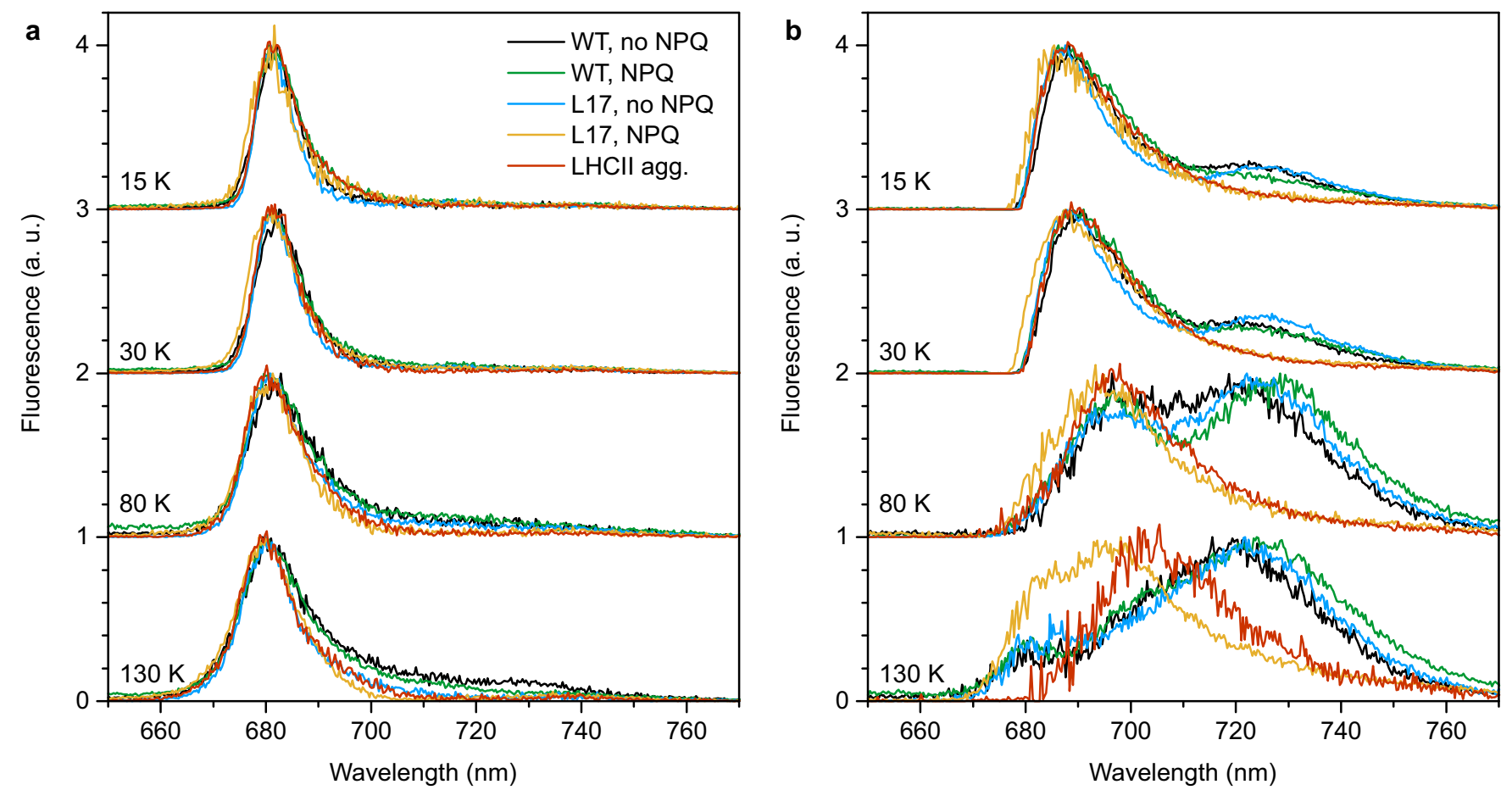

Figure S7. Fluorescence spectra of the 680-nm-emitting (a) and red-emitting (b) spectral components, obtained at various temperatures for the dark- and light- adapted chloroplasts from the lincomycin-pretreated wild-type and L17 mutant of the Arabidopsis thaliana (the same spectra as in Fig. S6). For comparison, the corresponding spectral components of LHCII aggregates from spinach ${ }^{7}$ are also shown (red lines).
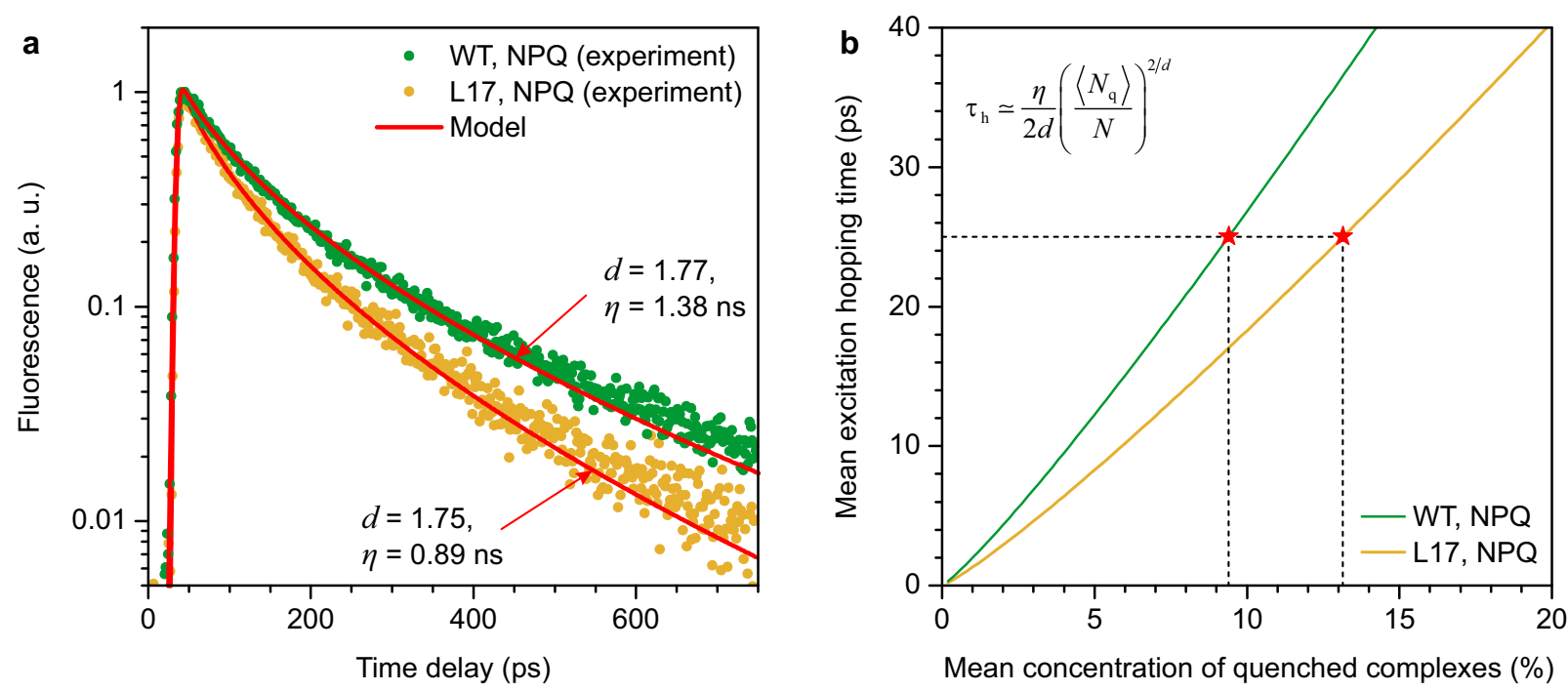

Figure S8. a, room-temperature fluorescence decay kinetics at $680 \mathrm{~nm}$ in the light-adapted chloroplasts from the lincomycin-pretreated WT and L17 Arabidopsis thaliana (dots) and their fits calculated using the fluctuating antenna model ${ }^{8}$ (red lines). The corresponding obtained model parameters are indicated for each kinetics. b, the obtained relationship between the mean inter-complex excitation hopping time, $\tau_{\mathrm{h}}$, and the mean relative number of the quenched complexes per antenna, $\left\langle N_{\mathrm{q}}\right\rangle / N$. Stars indicate the mean hopping time of $25 \mathrm{ps}$, corresponding to $\sim 9.5 \%$ and $13 \%$ of the quenched complexes in the light-adapted WT and L17 thylakoid membranes, respectively. 